\title{
PET/MRI: Paving the Way for the Next Generation of Clinical Multimodality Imaging Applications
}

\author{
Bernd J. Pichler ${ }^{1}$, Armin Kolb ${ }^{1}$, Thomas Nägele ${ }^{2}$, and Heinz-Peter Schlemmer ${ }^{3}$ \\ ${ }^{1}$ Laboratory for Preclinical Imaging and Imaging Technology of the Werner Siemens Foundation, Department of Radiology, \\ University of Tübingen, Tübingen, Germany; ${ }^{2}$ Department of Diagnostic and Interventional Neuroradiology, University of Tübingen, \\ Tübingen, Germany; and ${ }^{3}$ Department of Diagnostic and Interventional Radiology, University of Tübingen, Tübingen, Germany
}

Multimodality imaging and, more specifically, the combination of PET and CT has matured into an important diagnostic tool. During the same period, concepts for PET scanners integrated into an MR tomograph have emerged. The excellent soft-tissue contrast of MRI and the multifunctional imaging options it offers, such as spectroscopy, functional MRI, and arterial spin labeling, complement the molecular information of PET. The development of a fully integrated PET/MRI system is technologically challenging. It requires not only significant modifications of the PET detector to make it compact and insensitive to magnetic fields but also a major redesign of the MRI hardware.

Key Words: clinical multimodality imaging; PET; CT; PET/CT; arterial spin labeling (ASL); fMRI; spectroscopy; magnetic resonance tomograph (MRT); PET/MRI; positron emission tomography; MR/PET

J Nucl Med 2010; 51:333-336

DOI: 10.2967/jnumed.109.061853

Over the last 20 years, PET and MRI systems have evolved slowly but steadily. The photomultiplier-based PET detectors have remained more or less unchanged, ensuring stable operation and good signal performance despite bulk and high sensitivity to even the smallest magnetic fields. Improvements in PET technology were achieved with faster and low-noise electronics, faster and brighter scintillation crystals, optimized light-sharing schemes for the scintillation crystal arrangements, and smaller crystals (1). These adaptations led to PET scanners with whole-body scan times as short as $10 \mathrm{~min}$, yielding-together with improved reconstructions, attenuation- and scatter-correction algorithms-low-noise PET images. Advances paved the way to implementing the idea of time-of-flight PET in clinical scanners (2). Without a doubt, the most important step toward the establishment of PET as

Received Aug. 18, 2009; revision accepted Nov. 16, 2009.

For correspondence or reprints contact: Bernd J. Pichler, University of Tuebingen, Roentgenweg 11, Tuebingen, 72076 Germany.

E-mail: bernd.pichler@med.uni-tuebingen.de

COPYRIGHT (c) 2010 by the Society of Nuclear Medicine, Inc. a clinically viable tool was the introduction of combined PET/ CT in 1998 by David Townsend and Ronald Nutt (3-5). Nevertheless, many physicians of that time remained skeptical about the advantages of this dual-modality imaging system over stand-alone PET and CT.

Clinical MRI evolved toward higher fields, faster imaging sequences, and whole-body imaging capabilities. Especially for brain imaging, 3-T MRI is now the standard. Novel coil concepts combined with parallel acquisition techniques helped shorten examination times while maintaining high imaging quality (6).

\section{PET/MRI: TECHNICAL EVOLUTION}

The idea to combine PET and MRI arose as early as the mid 1990s, even before PET/CT was introduced. Simon Cherry and Paul Marsden saw the need for PET/MRI in small-animal imaging studies to add anatomic landmarks with high softtissue contrast to the molecular information delivered by PET (7). Preclinical PET/MRI work was followed by immediate commercial interest in combining PET and MRI, probably driven by the limited sensitivity of MRI to trace biomarkers or to reveal metabolites.

The PET/MRI combination requires 3 risky technologic steps that modify state-of-the-art PET and MRI. First, the photomultiplier technology must be replaced with magnetic field-insensitive avalanche photodiodes (8), which have been used in prototype animal PET systems that had a limited number of detectors and were temperature-controlled with costly cooling systems.

Second, compact PET detectors must be constructed to be invisible to the MRI and to not interfere with the field gradients or MR radiofrequency. Finally, the MRI scanner must be adapted to accommodate the PET detectors and to allow simultaneous data acquisition without mutual interference. The radiofrequency coils of the MRI scanner must be integrated into the PET system. Dedicated coils need to be built to minimize radiofrequency interference with the PET electronics and to avoid $\gamma$-ray scatter and attenuation. Furthermore, completely new strategies for PET attenuation correction, based solely on MRI information, have to be developed. The development of 
integrated PET/MRI is, therefore, a comprehensive endeavor that requires a significant advancement of PET detector technology, MRI system integration, and new software approaches.

Based on the technologic challenges to combine PET and MRI into a single gantry, Philips and Siemens proposed 2 fundamentally different prototype PET/MRI designs.

The Siemens prototypes include 4 dedicated brain PET scanners that slip-fit into a standard 3-T clinical MRI scanner (9). The PET detectors are based on avalanche photodiodes, lutetium oxyorthosilicate crystals, and modified electronic concepts yielding an overall good signal performance. Nevertheless, compared with state-of-the-art photomultiplier detectors, the prototypes have with 4 ns a reduced-by a factor of 5-coincidence-timing resolution. The PET provides a spatial resolution of $3 \mathrm{~mm}$ in full width at half maximum in iteratively reconstructed images. The hardware was engineered to limit mutual interference between the PET and the MRI. A major technologic challenge to using avalanche photodiodes is the temperature stabilization required to avoid significant gain drifts of $3.5 \% /$ Kelvin that result in imagenormalization artifacts, sensitivity variations, and, finally, PET quantification errors. The PET/MRI system, together with a dedicated radiofrequency head coil, allows simultaneous PET/MRI data acquisition of the human brain or body extremities. Therefore, these prototype scanners should be viewed as research tools for advanced brain imaging and as a first step toward a fully integrated whole-body system enabling testing of newly developed PET hardware, evaluation of novel multimodality imaging protocols, and implementation of MRI-based PET attenuation correction (10-12).

Philips developed a PET/MRI design in which the gantries are approximately $2.5 \mathrm{~m}$ apart but share a common patient handling system. This implementation does not allow for simultaneous data acquisition and, therefore, results in longer examination times. However, it enables the acquisition of spatially matched PET and MRI data to develop whole-body attenuation correction. Additionally, because some protocols and clinical cases may require that the MRI scan be performed during PET tracer uptake, the full advantage of PET/MRI can be explored for whole-body imaging. A clear constraint of this system is the lack of temporal matching between 2 functional datasets, such as for MRI perfusion or spectroscopy and PET tracer uptake.

\section{CLINICAL POTENTIAL OF PET/MRI}

The initial euphoria about the medical prospects of PET/ MRI is understandable $(9,13)$. Knowledge of functional anatomy is a key to medicine; therefore, methods for its assessment have been developed by physicians and physicists alike. Substantial progress has been made in noninvasive crosssectional imaging. To date, helical CT enables high-resolution anatomic imaging of the whole body within seconds. MRI yields superb soft-tissue contrast and functional information on perfusion, diffusion, or metabolism. PET enables physicians to visualize molecular tracers with picomolar sensitivity, providing information about cell metabolism and receptor status.
It is important to note that patient management in oncology is affected by PET/CT and MRI, as clinical studies and our daily clinical experience demonstrate. In fact, multimodality imaging is a fast-growing field in clinical practice, although, except for PET/CT, most examinations are performed on separate machines, a drawback that impacts daily routine: the process is time-consuming, expensive, and logistically demanding for patients and staff. Patient repositioning causes inaccurate anatomic matching, and side-by-side interpretation of images results in diagnostic inaccuracy. Software fusion of images is hampered by varying image properties such as spatial resolution, shifting, tilting, rotation, distortion, partial-volume effects, and nonrigid organ deformation. Finally, manipulating the vast amount of imaging — including multimodal—data and follow-up studies makes high demands on computer and software technology.

Indeed, PET/MRI has the potential to broaden our horizons in the emerging field of molecular imaging, because complementary anatomic and biologic information is obtained and synergisms of both modalities can be expected. Nevertheless, criticism is justified. The novel imaging technology may not enter clinical routine before its impact on diagnostic accuracy has been proven, and the effect on therapy management and cost-efficiency has been considered and validated. For example, it must be determined which unmet clinical needs can be addressed by PET/MRI, why higher diagnostic accuracy is anticipated compared with sequentially performed PET and MRI, and what the added value could be with respect to the established PET/CT technology. The high cost of integrated PET/MRI systems must be considered to establish what level of hardware integration is really mandatory to meet clinical requirements.

Capabilities and limitations of PET/MRI must be balanced and compared with PET/CT by considering technologic, scientific, medical, and economic aspects. It should not be forgotten that CT is a robust, widely distributed, and relatively inexpensive imaging modality that has evolved into a standard diagnostic tool in clinical practice. In combined PET/CT, cross-talk effects are virtually nonexistent and CT data can be used directly for the PET attenuation correction and image reconstruction. Clinical studies have demonstrated the advantages of PET/CT over separately performed PET and CT, and the technology has evolved rapidly into a powerful diagnostic tool, particularly in the field of oncology (14). Thus, PET and $\mathrm{CT}$ have already proven to be ideal partners.

However, from a clinical point of view, there are quite a few arguments for replacing CT with MRI. Whole-body MRI examinations that maintain state-of-the-art image quality for different body parts have recently become reality through technologic advances in multiple receiver and parallel-imaging technology (15). Furthermore, MRI applies only radiofrequency, and not ionizing irradiation, to the patient. The applied x-ray dose of a PET/CT scan predominantly depends on the CT examination protocol. The total exposure dose to a patient may be considerable, particularly when repeated whole-body examinations are required for therapeutic monitoring $(16,17)$. Reducing the radiation exposure is especially important for children and younger patients. 


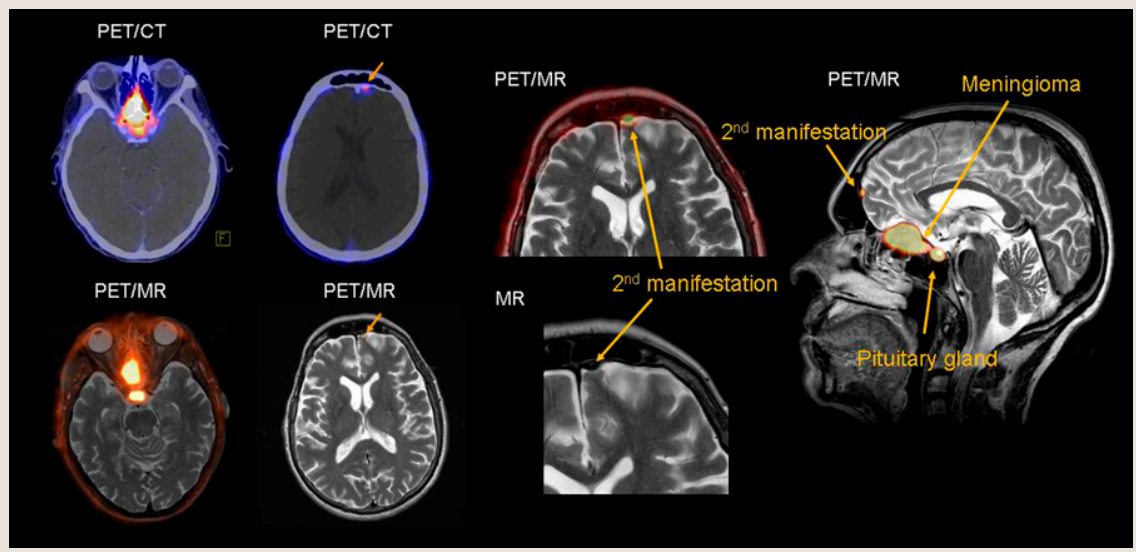

FIGURE 1. PET/CT and PET/MRI of 71-y-old woman with frontobasal meningioma in olfactory region. PET/CT images were acquired $20 \mathrm{~min}$ and $\mathrm{PET} / \mathrm{MR}$ images $100 \mathrm{~min}$ after injection of 135 MBq of ${ }^{68} \mathrm{Ga}-[1,4,7,10$-tetraazacyclododecane- $N, N^{\prime}, N^{\prime \prime}, N^{\prime \prime \prime}$-tetraacetic acid]-D$\mathrm{Phe}^{1}, \mathrm{Tyr}^{3}$-octreotide. Tracer uptake in the tumor is seen on PET images. In addition, second smaller and previously unknown frontal meningioma was seen on PET and possibly corresponded to small mass demonstrated on T2weighted turbo spin-echo MR images. This finding was not detected by CT.

It is reasonable to expect that brain PET/MRI will provide new insights in the field of neuroscience and neurologic disorders, such as neurodegeneration, brain ischemia, neurooncology, or seizures (18). It is feasible with current prototypes and future-generation systems to simultaneously study brain function, metabolism, oxygen consumption, and perfusion. The exact spatial and temporal coregistration of data will allow the attribution of functional and molecular information to even anatomically small brain structures. For the first time, it may become possible to study the correlation of local radiotracer uptake and brain perfusion. Time-dependant processes such as perfusion changes in stroke patients may rely on simultaneous diffusion-weighted imaging and detection of PET perfusion to determine the optimal therapy procedure. In neurooncology, an accurate spatial match between PET and MRI data is mandatory for both radiation therapy planning and biopsy guidance. PET may detect especially small lesions with higher sensitivity than MRI (Fig. 1). Figure 2 illustrates a clinical case of an anaplastic glioma that was missed on MRI and was described only incompletely on PET. The combination of PET and MRI revealed the full extent of the disease.

\section{WHOLE-BODY PET/MRI}

Whole-body PET/MRI will be of particular medical importance because systemic disorders such as cardiovascular disease and cancer increasingly account for morbidity and mortality. Therapeutic success with these chronic and often incurable diseases is linked to early diagnosis, accurate staging, and therapy monitoring. This requires repeated whole-body assessment of the extent of the disease, relapses, complications, and concomitant diseases. Clinical studies comparing ${ }^{18} \mathrm{~F}-\mathrm{FDG}$ PET/CT and whole-body MRI indicate that therapeutically relevant information is frequently obtained by PET or MRI but not necessarily by CT (19-23). For example, MRI is more sensitive than PET/CT in the detection of brain, bone, and liver metastases, whereas PET/CT is more accurate in the detection of lymph node metastases, characterization soft-tissue masses, and therapy monitoring (Fig. 3). The list of conceivable research topics and clinical applications is extensive. PET/MRI could be particularly useful for early tumor detection and functional therapy monitoring in oncology. It will likely be an ideal tool for investigating the effect of novel drugs, such as inhibitors of angiogenesis or modulators of the immune system. Integrated information on individual cell metabolism and microenvironment and their response to therapy will help elucidate the mechanism of action and optimize treatment schedules. Realtime monitoring of the success of radiofrequency ablation by PET may be an emerging application for PET/MRI (24).

Combining PET with cardiac MRI and whole-body MR angiography may enable detection and differentiation of vulnerable plaques. The combination of late-enhancement

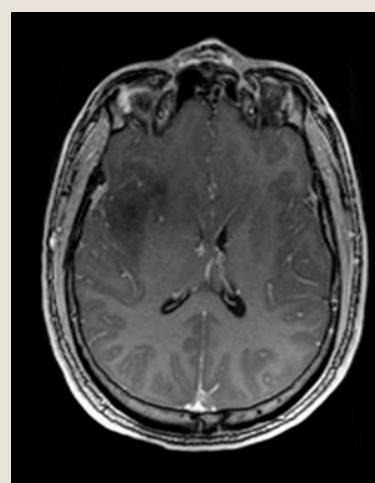

$\mathrm{T} 1+\mathrm{CA}$

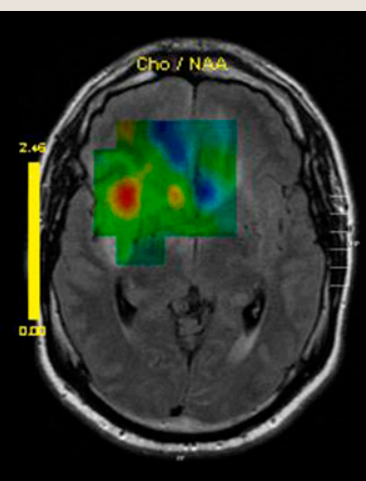

CSI Cho/NAA

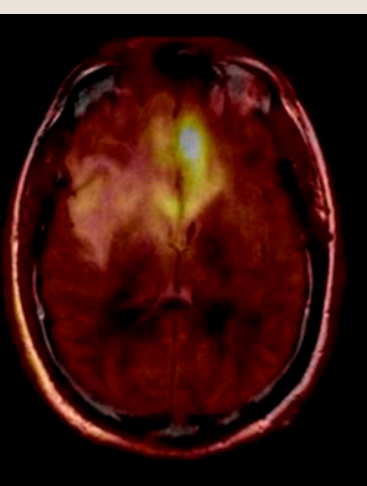

$\left[{ }^{11} \mathrm{C}\right] \mathrm{MET}-\mathrm{PET}$
FIGURE 2. A 36-y-old patient with brain gliomatosis was admitted for tumor biopsy. In addition to standard contrast-enhanced T1-weighted MRI (left), chemical shift imaging (CSI) (echo time $=135 \mathrm{~ms}$, center) and PET/MRI with ${ }^{11} \mathrm{C}$-methionine (right) were performed. On standard MRI, no contrast enhancement in any part of tumor was found, indicating low-grade tumor. On $\mathrm{CSI}$, mapping of choline/ $\mathrm{N}$-acetylaspartate (Cho/NAA) quotient showed hot spot in right insular region, whereas ${ }^{11} \mathrm{C}$-methionine uptake was most pronounced in basal frontal lobe on left

side. Because of discrepancy between CSI and PET/MRI, biopsy was performed in both locations and revealed anaplastic glioma (World Health Organization grade III) in frontal left region and low-grade glioma (World Health Organization grade II) in right insular region. $\mathrm{CA}=$ contrast agent. 


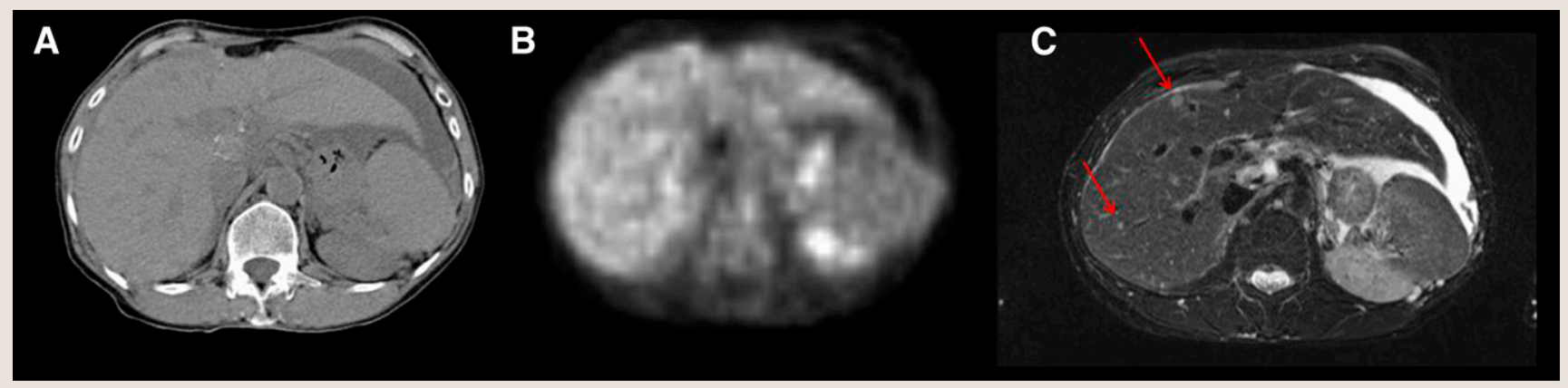

FIGURE 3. Abdominal unenhanced ${ }^{18} \mathrm{~F}-\mathrm{FDG}$ PET/CT and MRI of 55 -y-old woman with ovarian cancer showing liver metastases detected by MRI (C) but neither by PET (B) nor native CT (A). MRI is most sensitive for detecting small liver lesions because of its superb soft-tissue contrast, whereas PET and CT are limited because of lower contrast and physiologic ${ }^{18} \mathrm{~F}-\mathrm{FDG}$ liver uptake.

MRI and ${ }^{18}$ F-FDG uptake in a single imaging session may push cardiac imaging to another level (25). Simultaneously acquired PET and MRI data will allow accurate motion correction, particularly in cardiology but also in accurate detection of lesions in the abdomen or thorax. Disease- and therapy-specific PET/MRI examination protocols could be tailored to individual clinical conditions by using specific radiotracer and MRI sequence protocols.

\section{CONCLUSION}

Having witnessed an impressive technologic development of PET detector technology, first PET/MRI prototype systems, and MRI-based PET attenuation correction, as well as encouraging clinical and specifically preclinical PET/MRI results, we now seek opportunities to translate these technologic advances into clinical benefits.

\section{ACKNOWLEDGMENTS}

Part of this work was supported by the NIH (R21EB004483) and German DFG (PI771, SFB773).

\section{REFERENCES}

1. Pichler BJ, Wehrl HF, Judenhofer MS. Latest advances in molecular imaging instrumentation. J Nucl Med. 2008;49(suppl 2):5S-23S.

2. Karp JS, Surti S, Daube-Witherspoon ME, Muehllehner G. Benefit of time-offlight in PET: experimental and clinical results. J Nucl Med. 2008;49:462470 .

3. Mawlawi O, Townsend DW. Multimodality imaging: an update on PET/CT technology. Eur J Nucl Med Mol Imaging. 2009;36(suppl 1):S15-S29.

4. Beyer T, Townsend DW, Brun T, et al. A combined PET/CT scanner for clinical oncology. J Nucl Med. 2000;41:1369-1379.

5. Townsend DW, Beyer T. A combined PET/CT scanner: the path to true image fusion. Br J Radiol. 2002;75(spec no):S24-S30.

6. Wiesinger F, Van de Moortele PF, Adriany G, De Zanche N, Ugurbil K, Pruessmann KP. Potential and feasibility of parallel MRI at high field. NMR Biomed. 2006;19:368-378.

7. Shao Y, Cherry SR, Farahani K, et al. Simultaneous PET and MR imaging. Phys Med Biol. 1997;42:1965-1970.

8. Judenhofer MS, Wehrl HF, Newport DF, et al. Simultaneous PET-MRI: a new approach for functional and morphological imaging. Nat Med. 2008;14:459465 .
9. Schlemmer HP, Pichler BJ, Schmand M, et al. Simultaneous MR/PET imaging of the human brain: feasibility study. Radiology. 2008;248:1028-1035.

10. Hofmann M, Pichler B, Scholkopf B, Beyer T. Towards quantitative PET/MRI: a review of MR-based attenuation correction techniques. Eur J Nucl Med Mol Imaging. 2009;36(suppl 1):S93-S104.

11. Hofmann M, Steinke F, Scheel V, et al. MRI-based attenuation correction for PET/MRI: a novel approach combining pattern recognition and atlas registration. J Nucl Med. 2008;49:1875-1883.

12. Martinez-Moller A, Souvatzoglou M, Delso G, et al. Tissue classification as a potential approach for attenuation correction in whole-body PET/MRI: evaluation with PET/CT data. J Nucl Med. 2009;50:520-526.

13. von Schulthess GK, Schlemmer HP. A look ahead: PET/MR versus PET/CT. Eur J Nucl Med Mol Imaging. 2009;36(suppl 1):S3-S9.

14. Townsend DW. Dual-modality imaging: combining anatomy and function. J Nucl Med. 2008;49:938-955.

15. Schlemmer HP, Schafer J, Pfannenberg C, et al. Fast whole-body assessment of metastatic disease using a novel magnetic resonance imaging system: initial experiences. Invest Radiol. 2005;40:64-71.

16. Brix G, Lechel U, Glatting G, et al. Radiation exposure of patients undergoing whole-body dual-modality ${ }^{18}$ F-FDG PET/CT examinations. J Nucl Med. 2005; 46:608-613

17. Huang B, Law MW, Khong PL. Whole-body PET/CT scanning: estimation of radiation dose and cancer risk. Radiology. 2009;251:166-174.

18. Heiss WD. The potential of PET/MR for brain imaging. Eur J Nucl Med Mol Imaging. 2009;36(suppl 1):S105-S112.

19. Lauenstein TC, Freudenberg LS, Goehde SC, et al. Whole-body MRI using a rolling table platform for the detection of bone metastases. Eur Radiol. 2002;12:2091-2099.

20. Muller-Horvat C, Radny P, Eigentler TK, et al. Prospective comparison of the impact on treatment decisions of whole-body magnetic resonance imaging and computed tomography in patients with metastatic malignant melanoma. Eur $J$ Cancer. 2006;42:342-350.

21. Pfannenberg C, Aschoff P, Schanz S, et al. Prospective comparison of ${ }^{18} \mathrm{~F}$ fluorodeoxyglucose positron emission tomography/computed tomography and whole-body magnetic resonance imaging in staging of advanced malignant melanoma. Eur J Cancer. 2007;43:557-564.

22. Schmidt GP, Baur-Melnyk A, Herzog P, et al. High-resolution whole-body magnetic resonance image tumor staging with the use of parallel imaging versus dual-modality positron emission tomography-computed tomography: experience on a 32-channel system. Invest Radiol. 2005;40:743-753.

23. Antoch G, Vogt FM, Freudenberg LS, et al. Whole-body dual-modality PET/CT and whole-body MRI for tumor staging in oncology. JAMA. 2003;290:31993206.

24. Bao A, Goins B, Dodd GD III, et al. Real-time iterative monitoring of radiofrequency ablation tumor therapy with ${ }^{15} \mathrm{O}$-water PET imaging. $\mathrm{J} \mathrm{Nucl}$ Med. 2008;49:1723-1729.

25. Nekolla SG, Martinez-Moeller A, Saraste A. PET and MRI in cardiac imaging: from validation studies to integrated applications. Eur J Nucl Med Mol Imaging. 2009;36(suppl 1):S121-S130. 